\section{A S E}

OF

\section{IUMBAR ARTIFICIAL ANUS TERMINATING SUCCESSFULLY.}

\section{By M. Amussat.}

In a recent number of the "Provincial Journal" we published a highly interesting case of lumbar artificial anus, performed at Leeds, by Mr. Teale. The operation was not successful, probably because it had been delayed too long. Since then M. Amussat has published the following successful case, which makes the sixth performed by him. We insert it, slightly abridged, as we are desirous of directing the attention of English surgeons to an operation which has not, we fear, received from them the degree of attention that it merits.

M. C-, tailor, 57 years of age, was attacked by pleurisy at the age of 17 ; since then he has been subject to cough and frequent spitting of blood, which always made him dread consumption, but no sign of this disease has appeared. For a length of time, whenever fatigued, he felt a dull, deep pain near the spleen, which compelled him to suspend his work. About nine months ago the patient first perceived a difficulty in passing his stools, which were small in quantity, of a greenish tint, and often tinged with blood; afterwards he passed nearly a glassful of pure blood by the ants. These symptoms were relieved by appropriate treatment, but soon afterwaras they returned, and the patient perceived a tumour in the left iliac fossa; this was the seat of considerable lancinating pain, and all the symptoms were aggravated from this time. The patient now came to Paris for advice. On examination a tumour was distinctly felt in the left iliac fossa, appearing to rest on and following the course of the sigmoid flexure of the colon; it was from two and a half to three inches in length, by one thick, and it was impossible to say whether or not it was formed by the intestine. Various purgative remedies were now administered, but without effect; and the abdomen became tympanitic and painful. M. Rostan, who was consulted, diagnosticated a cancerous tumour of the intestine, and thought that the patient's death was not far distant. On the following day, November 20, 1841, M. Amussat was consulted; the abdomen was now greatly distended with gaz, and there was frequent inclination to vomit, with some hiccup; the patient passed a good deal of air from the mouth, but none per anum. M. Amussat agreed in opinion with $M$. Rostan, but was desirons of having further advice; in the mean time purgatives were given, and forced enemata were thrown up without effect.

21. The patient passed a very bad night; the tympanitis continued to extend; towards morning he vomited some mucus mixed with blood. Four forced enemata were carefully thrown up, and probably passed beyond the obstruction in the intestine, for they did not return; from this period all the symptoms were decidedly aggravated; the patient felt as if he were suffocating, and loudly demanded the operation as a means of relief. In consultation with MM. Rostan and Breschet the operation was decided upon, but during the night the patient vomited a large quantity of mucous fluid mixed with blood. This led to some discussion between the medical men on the following day. It was thought that the blood vomited might be simply an exhalation from the mucous membrane of the stomach or bowels; but on the other hand that it might indicate a cancerous disease of the pylorus, and hence that an operation was contraindicated.

During the 22nd the patient felt better; he was less oppressed, and seemed relieved by the vomiting ; in the evening a few small enemata had been thrown up, and brought away a small quantity of fœcal matter.

On the 23rd, M. Magendie joined the other medical men in consultation; he thought that, as the patient's condition was somewhat improved, and the obstruction of the bowels not complete, it would be better to defer the operation; he recommended a continuation of the means already employed, and that an cesophagus tube should be passed up as far as the obstruction, with the hope of overcoming it ; in one case which had occurred in his wards at the Hotel-Dieu, and where a cancer of the sigmoid flexure of the colon had been diagnosticated, the tube had succeeded in removing the obstruction. M. Breschet also thought that the operation should be deferred.

M. Rostan said that, as he believed there was a cancerous disease of the colon, he did not think there was another one in the stomach; in the numerous autopsies that he had made, he never found cancerous deposit in the stomach and in the the time. He considered the blood vomited to have been a mere exhalation, and did not think that the operation was contraindicated by this circumstance.

As opinions were thus divided, it was determined to delay the operation. In the evening, M. Amussat passed up an cesophagus bougie, at the extremity of which was a small wax bougie, but he was unable to pass the obstruction; on withdrawing it, the patient felt an inclination to go to stool, and passed about a spoonful of fæcal matter; for the last twenty days he never passed any thing without the aid of enemata.

24. The patient again passed some fœces with the assistance of a lavement; the abdomen is softer, and there is no sense of suffocation as before. During the next three days things remained in the same state.

28. The patient slept a little during the night, but he is depressed in spirits; the abdomen is greatly distended with gaz; and for the last thirty-six hours he has not passed any wind or fœcal matter through the anus. An œsophagus bougie was now passed up to a distance of eleven inches; on withdrawing it, the end was seen to be strongly bent on itself and tinged with bloody mucus; some gaz came away without any relief.

29. The tube was again introduced, and seemed to have passed the obstruction, for it entered to a depth of thirteen inches; a clyster was immediately thrown up without effect. Two forced enemata were now thrown up, and immediately passed with a considerable quantity of fœcal matter.

On the 2nd of December the tube was passed up to a depth of sixteen inches, and a lavement injected, but hardly a trace of fæces came away; from this time the evacuations were completely suppressed, 
and the patient insisted on having the operation performed on the 8th of December, 1841 .

It was decided that the opening should be made in the right lumbar region, on account of the presumed disease at the left side. M. Amussat made a transverse incision midway between the crista ilii and the last rib, and then divided the subjacent tissues, until a fold of intestine was seen; this was enveloped in fatty tissue, and an artery ran close upon it ; the vessel, which had been accidentally divided, was twisted. Here, however, a great difficulty presented itself-Was the intestine, seen at the bottom of the wound, a portion of the colon or of the small intestines? In this uncertainty nothing remained but to expose the muscular coat of the gut, and trust to chance; a needle armed with a ligature was passed through the intestine, and immediately some gaz, having a fœcal odour, escaped. A tenaculum was now fixed close to the point where the ligature had been applied, and the intestine being steadied by these means, a small incision was made with the scissors; this was enlarged with a probe-pointed bistoury, and the finger then introduced into the gut; on withdrawing the finger, a considerable quantity of gaz and fœcal matter escaped. Three enemata were now injected into the artificial opening and came away, bringing with them three washhand basinsful of fæces, containing the seeds of grapes and pears, which the patient had eaten about two months previously.

The intestine was fixed to the anterior angle of the wound by four points of suture ; the pationt folt immediate relief from the operation; the size of the abdomen was considerably reduced, and the breathing was much freer.

It is unnecessary to transcribe the daily reports of the case; on the the 14th (7th day), M. Amussat divided the sutures by which the intestine was fixed to the wound ; the patient was allowed to have a little broth.

On the 10th day he passed a very copious stool, hard and cylindrical, through the anus, a circumstance which showed that the intestinal obstruction was not complete; on the 13th day the patient was able to come down stairs from the sixth story of the house in which he lodged. From this period to the 50th day after the operation the patient continued to pass his evacuations through the artificial opening; but a quantity of muco-purulent matter was daily discharged through the anus.

On the 50th day the patient returned to his family in the country, having made a journey of thirty-nine leagues without any inconvenience.

On the 22nd of February, 1842, M. Amussat heard from his medical attendant that he continued in an excellent state, but that occasionally fetid purulent matter was dischaged through the anus.

The object, then, of the operation in this case, was fully attained. During forty days the obstacle to the evacuation of the fæces was so great that the patient was reduced to imminent danger, and would, in all probability, have speedily perished; he has now a chance of living for a considerable time.

Since the operation just described, M. Amussat performed a sixth of the same kind on an infant affected with congenital imperforation of the rectum. $M$. Amussat had tried to open the rectum through the anus, but was unable to find the gut; he then made an artificial opening in the left lumbar region and the infant, now two months old, is well. This is the sixth successful case which has occurred in the practice of M. Amussat.-Gaz des Hop., No. 37.

\section{ON THE}

\section{OPERATION OF TRACHEOTOMY IN CROUP,}

PERFOREED AT

\section{THE CHILDREN'S HOSPITAL,}

\author{
AND ON ITS RESULTS.
}

By A. Becquerex, M.D.

During the year 1841, twenty cases of true croum were admitted into the Children's Hospital, Paris The whole of these patients died; nineteen perished shortly after the attack, and one who had recovered from the croup died in two months afterwaxds of pulmonary tubercles. Of the twenty cases, thirteen were boys and seven girls; the ages of the patients varied from two to fourteen years.

The operation of tracheotomy was pexformed in nine cases, eight times by the hospital internes, and once by $\mathbf{M}$. Guersant, jun. In the following remans I shall endeavour to describe, first, the circumstances under which the operation was performed; second, its immediate results; and third, its remoto consequences.

\section{Circumstances under which the poration wan Performed.}

In all the nine cases the children were in the most dangerous state, and no other hope of safety remained; in one, indeed, the operation was performed at the instant the little patient expired, and with the faint hope of restoring it to life. The subject of anether case was a child four years of age, who had been ill three days; the cough and voice wore extinct; and asphyxia was imminent; there were several traces of false membranes on the amygdalæ and pharynx. The child was carried to the hospital, and operated on with out delay. The symptoms of asphyxia, howerer, were much aggravated during her removal, and the patient expired immediately after the operation. On examination a cylinder of false membrane was found adhering to the larynx and two upper thirds of the trachea. Had the operation been performed half an hour earlier, and the false membrane been expelled, it is clear that the operation might have been succeseful. In a third case, M. Guersant, jun., operated on a chitd affected with stridulous laryagitis which bore a pezfoct resemblance to true croup ; but no trece of nlocenbrane escaped from the opening or wra found after death. The periods at which tracheotomy was performod in the nine cases were as follows: -1 on the second day; 3 on the third; 3 on the fourth; 1 on the fifth; and 1 on the sixth day.

\section{Immediate Results of the Operation.}

In none of the cases did any blood penetrate into the trachea, in sufficient quantity to give rise to or increase the asphyxia. The rperation was eary of execution and quickly performed. 